\title{
The association between cognitive function and white matter lesion location in older adults: a systematic review
}

\author{
Niousha Bolandzadeh", Jennifer C Davis², Roger Tam ${ }^{3}$, Todd C Handy ${ }^{4}$ and Teresa Liu-Ambrose ${ }^{1,5,6^{*}}$
}

\begin{abstract}
Background: Maintaining cognitive function is essential for healthy aging and to function autonomously within society. White matter lesions (WMLs) are associated with reduced cognitive function in older adults. However, whether their anatomical location moderates these associations is not well-established. This review systematically evaluates peer-reviewed evidence on the role of anatomical location in the association between WMLs and cognitive function.
\end{abstract}

Methods: In accordance with the preferred reporting items for systematic reviews and meta-analysis (PRISMA) statement, databases of EMBASE, PUBMED, MEDLINE, and CINAHL, and reference lists of selected papers were searched. We limited our search results to adults aged 60 years and older, and studies published in the English language from 2000 to 2011. Studies that investigated the association between cognitive function and WML location were included. Two independent reviewers extracted: 1) study characteristics including sample size, sample characteristic, and study design; 2) WML outcomes including WML location, WML quantification method (scoring or volume measurement), strength of the MRI magnet in Tesla, and MRI sequence used for WML detection; and 3) cognitive function outcomes including cognitive tests for two cognitive domains of memory and executive function/processing speed.

Results: Of the 14 studies included, seven compared the association of subcortical versus periventricular WMLs with cognitive function. Seven other studies investigated the association between WMLs in specific brain regions (e.g., frontal, parietal lobes) and cognitive function. Overall, the results show that a greater number of studies have found an association between periventricular WMLs and executive function/processing speed, than subcortical WMLs. However, whether WMLs in different brain regions have a differential effect on cognitive function remains unclear.

Conclusions: Evidence suggests that periventricular WMLs may have a significant negative impact on cognitive abilities of older adults. This finding may be influenced by study heterogeneity in: 1) MRI sequences, WML quantification methods, and neuropsychological batteries; 2) modifying effect of cardiovascular risk factors; and 3) quality of studies and lack of sample size calculation.

Keywords: White matter lesions, Distribution, Cognition, Aging

\footnotetext{
* Correspondence: tlambrose@exchange.ubc.ca

'Department of Physical Therapy, University of British Columbia, 212-2177

Wesbrook Mall, Vancouver, BC V6T 1Z3, Canada

${ }^{5}$ Brain Research Centre, University of British Columbia, 212-2177 Wesbrook

Mall, Vancouver, BC V6T 1Z3, Canada

Full list of author information is available at the end of the article
} 


\section{Background}

The world's population is aging [1]. Maintaining cognitive function is essential for healthy aging and to function autonomously within society.

With age, the brain undergoes both structural and functional changes [2-5]. Specifically, cerebral white matter lesions (WMLs) are prevalent among adults aged 60 years or older [6,7]. These lesions are due to damage to the brain parenchyma [8], ranging from demyelination to complete axonal disruptions $[9,10]$. Although their pathogenesis is unknown, there is a growning recognition that WMLs are most likely the result of cerebrovascular disorders and cerebral ischemia [8,11-13]. The current gold standard for diagnosis of WMLs includes various MRI sequences, such as $\mathrm{T} 1, \mathrm{~T} 2$, proton density (PD), or fluid attenuated inversion recovery (FLAIR).

White matter lesions are associated with both impaired mobility and reduced cognitive performance as measured by standard neuropsychological testing, which might be caused by impairing the speed or integrity of signal transmission $[14,15]$. Specially, WML load has a negative impact on multiple domains of cognitive function such as memory, processing speed, attention, and executive function $[8,16]$. Pantoni et al. [16] summarized the results of 16 studies focusing on the effect of WMLs on different cognitive domains. Their results showed that, despite the fact that the probability of finding a positive association between WML load and cognitive decline may be affected by the cognitive domains assessed, an effect of WML on cognition was present invariably. However, emerging evidence suggests that WML distribution, as well as load, may also be a predictor of reduced cognitive performance $[17,18]$. In a study by Kim et al. [17], it is suggested that a specific distribution of fiber tract damage is more associated with cognitive and motor impairment, compared with the total WML load. Thus, we conducted a systematic review to ascertain the role of anatomical location in the association between WMLs and cognitive function in older adults.

\section{Methods}

\section{Search strategy}

In accordance with the preferred reporting items for systematic reviews and meta-analysis (PRISMA) statement [19], we [NB, JCD and TLA] conducted a search of EMBASE, MEDLINE, PUBMED, and CINAHL supplemented by manual search of included articles' reference lists. The search strategy (Figure 1(A)) was developed by April $19^{\text {th }}$ 2011, and includes studies from 2000 to 2011. We limited our search results to adults aged 60 years and older, and studies published in the English language.

\section{Study selection}

We excluded case-studies, reviews, and articles lacking WML quantification or measurements of cognitive function, based on their titles and abstracts (Figure 1(B)).
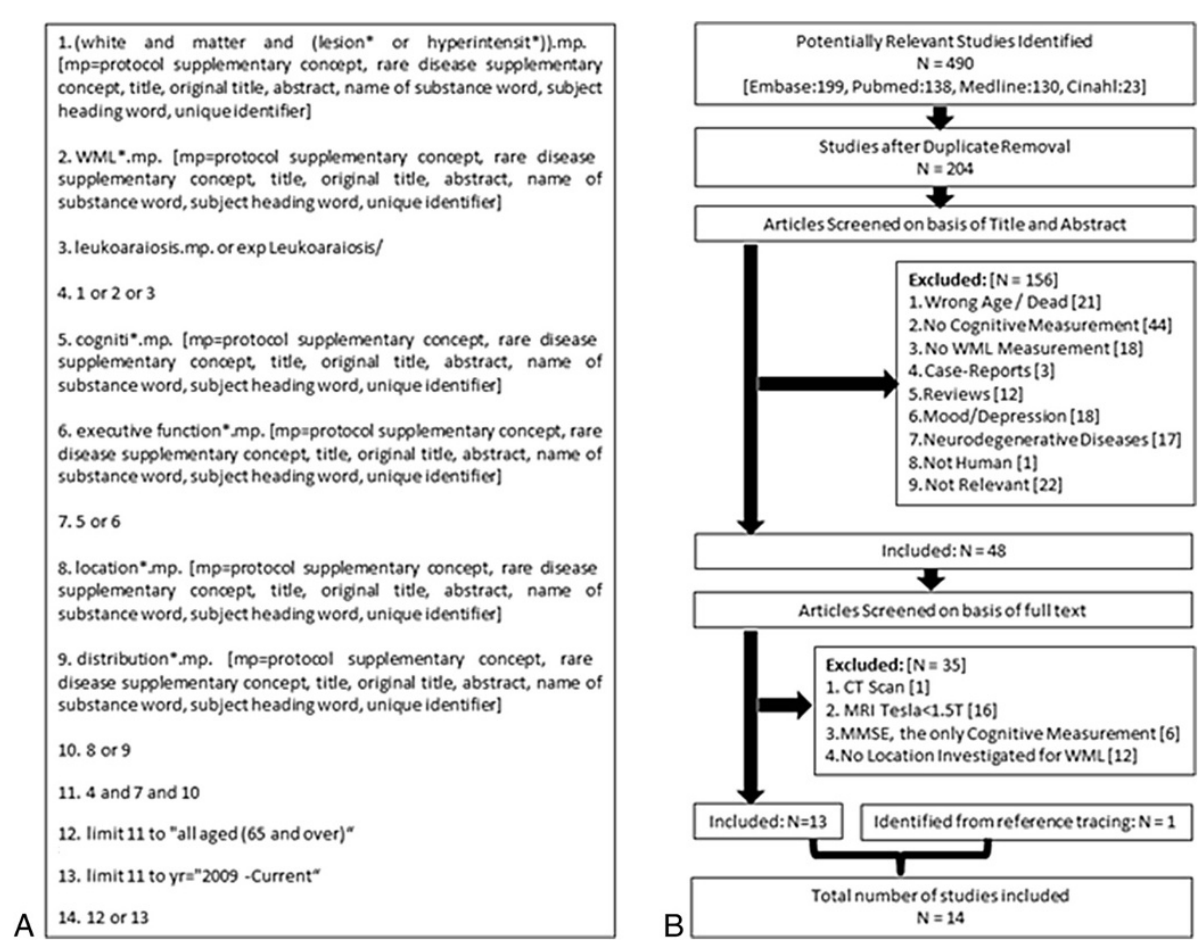

Figure 1 (A): Searching strategy retrieved from Ovid, (B): Flowchart of study selection. 
Also, any study with the primary focus on psychiatric conditions (e.g., depression) or progressive neurodegenerative diseases (except for Alzheimer's disease (AD) and cerebrovascular disorders due to the high prevalence of WMLs) was excluded. Based on full text review, we excluded studies that: 1) used computed tomography (as it is less sensitive than MRI in detection of WMLs [20]), or used MRI device with a magnet strength of less than $1.5 \mathrm{~T}$ and; 2 ) assessed only global cognition (measured by mini-mental state examination (MMSE)) as it may not be sensitive to the differential effects of WML location; and 3) did not detail WML location.

\section{Data extraction and quality assessment}

We [NB and TLA] developed a list of extraction items including: 1) study characteristics; 2) WML outcomes; and 3) cognitive function outcomes. One study [21] did not report the strength of MRI magnet and NB contacted the author.

Two authors [NB, TLA] independently evaluated each study based on four quality assessments questions (see Table 1), and all the discrepancies were reviewed by JCD and RT. Assessing the validity of WML quantification was influenced by the difficulty in the differential diagnosis of WMLs, which requires expert radiological knowledge to be done accurately [22]. In addition, the intensity range of lesions typically overlaps with those of healthy tissues, so automatic identification methods tend to produce more false positives as compared with manual identification by a radiologist [23]. Therefore, our assessment favors quantification methods that use radiologist/physician identification of WMLs. We used dichotomized answers (+: yes, -: no) for the quality assessment questions.

\section{Results}

\section{Overview of studies}

The initial number of articles identified was 490 (Figure 1(B)). After duplicate removal, 156 papers were further excluded using their title and abstract. We conducted a full text review of the remaining 48 articles. In total, 14 articles met the inclusion criteria (see Tables 2, 3, 4, 5). These articles were further categorized into two groups based on the cognitive status of their study samples: 1) studies that did not compare subjects based on cognitive status (i.e., normal, cognitively impaired but not demented, and demented); and 2) studies that classified and compared subjects based on cognitive status. Table 6 shows the most commonly-used cognitive tests in the 14 included studies.

\section{Studies that did not compare subjects based on cognitive} status

Subcortical vs. periventricular WML

Five studies [24-28] - four cross-sectional studies and one prospective study - compared the association of subcortical versus periventricular WMLs with cognitive function. In the first cross-sectional study of 1077 older adults [24], WMLs were defined as T2 and PD hyperintensities that were not T1 hypointensities. Four lobes of frontal, parietal, occipital, and temporal were considered for subcortical WML scoring. Three regions adjacent to frontal horns, lateral ventricles wall, and occipital horns were selected for periventricular WML scoring. The

Table 1 Quality assessment results for included studies

\begin{tabular}{|c|c|c|c|c|}
\hline Reference & $\begin{array}{l}\text { Q1. Was the WML } \\
\text { identification done by } \\
\text { a radiologist/physician? }\end{array}$ & $\begin{array}{l}\text { Q2. Was the cognitive } \\
\text { performance measured } \\
\text { using a standardized method? }\end{array}$ & $\begin{array}{l}\text { Q3. Was there } \\
\text { a sample size } \\
\text { calculation? }\end{array}$ & $\begin{array}{l}\text { Q4. Were age or } \\
\text { education considered } \\
\text { as confounders? }\end{array}$ \\
\hline Groot et al. et al. [24] & + & + & - & + \\
\hline Shenkin et al. [25] & + & + & - & - \\
\hline Baune et al. [26] & - & + & - & + \\
\hline Kim et al. [27] & - & + & - & + \\
\hline Silbert et al. [28] & - & + & - & + \\
\hline McClleland et al. [21] & + & + & - & + \\
\hline Wright et al. [29] & - & + & - & + \\
\hline Kaplan et al. [30] & - & + & - & + \\
\hline Wakefield et al. [31] & - & + & - & + \\
\hline O'Brien et al. [32] & + & + & - & + \\
\hline Smith et al. [14] & - & + & - & + \\
\hline Burns et al. [33] & + & + & - & + \\
\hline Ishii et al. [34] & + & + & - & + \\
\hline Tullberg et al. [35] & - & + & - & - \\
\hline
\end{tabular}


Table 2 Characteristics of studies included in this systematic review

\begin{tabular}{|c|c|c|}
\hline Reference & Sample size & Study design \\
\hline Publishing year & Sample characteristics & \\
\hline Groot et al. [24] & 1077 & Cross-Sectional \\
\hline 2000 & $\begin{array}{l}\text { Subsample of Rotterdam } \\
\text { and Zeotemeer Studies }\end{array}$ & \\
\hline
\end{tabular}

Shenkin et al. [25] 105

Cross-Sectional

2005

Random Sample of

Community-Dwelling

Participants

Baune et al. [26]

268

2009

Subsample of MEMO Study

Kim et al. [27]

84

2011

Random Sample of

Normals/Recruited from

Memory Clinic

\begin{tabular}{|c|c|c|}
\hline Silbert et al. [28] & 104 & Longitudinal \\
\hline 2008 & $\begin{array}{l}\text { Subsample of Oregon Brain } \\
\text { Aging Study }\end{array}$ & \\
\hline McClleland et al. [21] & 3647 & Cross-Sectional \\
\hline 2000 & Subsample of CHS Cohort & \\
\hline Wright et al. [29] & 656 & Cross-Sectional \\
\hline 2008 & $\begin{array}{l}\text { Subsample of NOMAS Cohort } \\
\text { study }\end{array}$ & \\
\hline Kaplan et al. [30] & 95 & Cross-Sectional \\
\hline 2009 & Random Sample of Participants & \\
\hline Wakefield et al. [31] & 99 & Cross-Sectional \\
\hline 2010 & $\begin{array}{l}\text { Sample Selected for a } \\
\text { Longitudinal Study }\end{array}$ & \\
\hline O'Brien et al. [32] & 149 & Cross-Sectional \\
\hline 2002 & Subsample of SCOPE Study & \\
\hline Smith et al. [14] & 145 & Cross-Sectional \\
\hline 2011 & $\begin{array}{l}\text { Subsample of Prospective } \\
\text { Study }\end{array}$ & \\
\hline Burns et al. [33] & 156 & Cross-Sectional \\
\hline 2005 & $\begin{array}{l}88 \text { Normal }(C D R=0), 68 \\
\text { Early-Stage } A D(C D R=0.5,1)\end{array}$ & \\
\hline Ishii et al. [34] & 453 & Cross-Sectional \\
\hline 2007 & $340(C D R=0), 113(C D R=0.5)$ & \\
\hline Tullberg et al. [35] & 78 & Cross-Sectional \\
\hline 2004 & $\begin{array}{l}22 \text { Normal }(C D R=0), 30 \text { CIND } \\
(C D R=0.5), 26 \text { Demented } \\
(C D R \geq 1)\end{array}$ & \\
\hline
\end{tabular}

Abbreviations: $M E M O=$ Memory and Morbidity in Augsburg Elderly; $C D R=$ Clinical Dementia Rating Scale; CHS = Cardiovascular Health Study; NOMAS = Northern Manhattan Study; SCOPE = Study on Cognition and Prognosis in Elderly; $C I N D=$ Cognitively Impaired not Demented.

neuropsychological battery evaluated two domains of memory and executive function/processing speed. The results showed that when controlled for subcortical WML severity, increased periventricular WML severity in all the three regions was associated with reduced performance in both cognitive domains $(\mathrm{p}<0.01)$. However, when controlled for periventricular WMLs, no such association was found for subcortical WMLs.

In the second cross-sectional study of 105 older adults [25], WMLs were identified using T2 and FLAIR scans. Results showed that higher periventricular and subcortical WML scores were not significantly associated with reduced memory and executive function/processing speed.

In a sample of 268 older adults [26], WMLs were categorized into three groups of: 1) large subcortical WMLs defined as PD and T2 hyperintensities that were not T1 hypointensities; 2) infarction lesions defined as lesions of $\geq 2 \mathrm{~mm}$ that were either T2 hyperintensities, or PD and T1 hypointensities; and 3) periventricular WMLs. The results indicated that large subcortical WMLs were significantly associated with memory, and infarction lesions were significantly associated with executive function/ processing speed $(\mathrm{p}<0.05)$. Contrary to the results of two previously mentioned studies, this study found no significant relationship between periventricular WMLs and cognitive performance.

In the last cross-sectional study, Kim et al. [27] defined WMLs as T2 and FLAIR hyperintensities. Over the 84 older adults, only periventricular WML was significantly correlated with memory and executive function/processing speed, when both the periventricular and subcortical WMLs were entered simultaneously into the regression model $(\mathrm{p}<0.05)$.

The one longitudinal study [28] used a sample of 104 subjects to investigate the impact of WML volume progression on the rate of cognitive decline. White matter lesions were defined as PD and T2 hyperintensities. Infarction lesions - detected by their clean or sharp edges, and if they were relatively dark on PD scans - were excluded from WML analysis. The neuropsychological battery assessed only memory. Higher rate of subcortical (but not periventricular) WML volume change was associated with increased rate of decline in memory scores $(\mathrm{p}<0.001)$.

\section{Regional WMLs}

Six cross-sectional studies [14,21,29-32] examined the association between WMLs in specific brain regions (e.g., frontal, parietal, etc.) and cognitive performance. McClelland et al. [21] defined WMLs as PD and T2 hyperintensities that were $\mathrm{T} 1$ hypointensity. The results in 3647 older adults suggested that WMLs located in cerebellar and cerebral white matter and basal ganglia were significantly associated with reduced processing speed performance $(\mathrm{p}<0.05)$.

Among 656 older adults, Wright et al. [29] differentiated subclinical infarction lesions from the rest of 
Table 3 Outcome Measures: white matter lesion quantification

\begin{tabular}{|c|c|c|c|}
\hline Reference & Sequence & WML Location & MRI \\
\hline WML Type & $\overline{\text { WML Quantification }}$ & & Magnet \\
\hline Groot et al. [24] & $\mathrm{PD}, \mathrm{T} 1, \mathrm{~T} 2$ & S: Four lobes of Frontal, Parietal, Occipital, and Temporal & $1.5 \mathrm{~T}$ \\
\hline P, S, Regions & Scoring & P: Adjacent frontal horns, lateral ventricles wall, and occipital horns & \\
\hline Shenkin et al. [25] & T2, FLAIR & - & $1.5 \mathrm{~T}$ \\
\hline$S, P$ & Scoring & & \\
\hline Baune et al. [26] & $\mathrm{PD}, \mathrm{T} 1, \mathrm{~T} 2$ & - & $1.5 \mathrm{~T}$ \\
\hline$S, P$ & Scoring & & \\
\hline Kim et al. [27] & T2, FLAIR & - & $1.5 \mathrm{~T}$ \\
\hline S, P & Volum & & \\
\hline Silbert et al. [28] & $P D, T 2$ & - & $1.5 \mathrm{~T}$ \\
\hline$S, P$ & Volume & & \\
\hline McClleland et al. [21] & $\mathrm{PD}, \mathrm{T} 1, \mathrm{~T} 2$ & Cerebral White Matter, Cerebellar White Matter, Basal Ganglia & $1.5 \mathrm{~T}$ \\
\hline Regions & Scoring & & \\
\hline Wright et al. [29] & PD, T2, FLAIR & Frontal, Deep, and Occipital-Temporal-Parietal & $1.5 \mathrm{~T}$ \\
\hline S, I, Regions & Volume & & \\
\hline Kaplan et al. [30] & T2, FLAIR & Frontal and Posterior Regions & $3.0 \mathrm{~T}$ \\
\hline Regions & Volume & & \\
\hline Wakefield et al. [31] & T1, FLAIR & Anterior, Superior, Posterior Corona Radiata & $3.0 \mathrm{~T}$ \\
\hline Regions & Volume & Cingulate Gyrus, Genu, Body, Splenium of Corpus Callusum & \\
\hline & & Anterior and Posterior Limb of Internal Capsule & \\
\hline & & Superior Longitudinal Fasciculus & \\
\hline O'Brien et al. [32] & T2, FLAIR & Internal and External Capsule & $1.5 \mathrm{~T}$ \\
\hline Regions & Scoring & & \\
\hline Smith et al. [14] & $P D, T 1, T 2$ & Whole Brain & $1.5 \mathrm{~T}$ \\
\hline Regions & Volume & & \\
\hline Burns et al. [33] & $\mathrm{T} 1, \mathrm{~T} 2$ & S: Frontal, Parietal, Temporal, and Occipital Lobes & $1.5 \mathrm{~T}$ \\
\hline $\mathrm{S}, \mathrm{P}$, Regions & Scoring & P: Right and Left Frontal Horns, Posterior Horns, and Ventricular Bodies & \\
\hline Ishii et al. [34] & $\mathrm{T} 2$ & S: Left and Right & $1.5 \mathrm{~T}$ \\
\hline P, S, Regions & Scoring & P: Anterior and Posterior & \\
\hline Tullberg et al. [35] & $\mathrm{T} 1, \mathrm{~T} 2$ & Orbitofrontal, Prefrontal, Dorsolateral Frontal, Parietal, and Occipitotemporal & $1.5 \mathrm{~T}$ \\
\hline Regions & Volume & & \\
\hline
\end{tabular}

Abbreviations: $P D=$ Proton Density; FLAIR = Fluid Attenuated Inversion Recovery.

WMLs based on the size, location, and imaging characteristics obtained from PD, T2, and FLAIR scans. They were grouped by location into frontal, deep and occipital-temporal-parietal networks. The neuropsychological battery assessed only executive function/processing speed. The results demonstrated that individuals with infarction lesions in frontal and deep locations had significantly worse cognitive performance $(\mathrm{p}<0.05)$.

Kaplan et al. [30] studied a sample of 95 older adults. White matter lesions were defined as FLAIR and T2 hyperintensities, and were categorized into frontal and posterior regions. The results showed that frontal WMLs were associated with memory $(\mathrm{p}<0.05)$ and executive function/processing speed $(\mathrm{p}<0.001)$.
Furthermore, Wakefield et al. [31] detected WMLs based on FLAIR and T1 scans in a sample of 99 community-dwelling older adults. The following regions of interest were segmented for WMLs: anterior, superior, and posterior corona radiata; cingulate gyrus, genu, body, and splenium of corpus callusum; anterior and posterior limb of internal capsule; and superior longitudinal fasciculus. The neuropsychological battery assessed only executive function/processing speed. In regions of posterior corona radiata and splenium of corpus callosum, the total amount of WMLs was significantly associated with executive function/processing speed $(\mathrm{p}<0.05)$.

O'Brien et al. [32] detected WMLs based on FLAIR and T2 scans, in 149 older adults. The focus of their 


\begin{tabular}{|c|c|c|}
\hline Reference & Executive function / Processing speed & Memory \\
\hline Groot et al. et al. [24] & $\begin{array}{l}\text { Stroop, Letter-Digit Substitution Task, } \\
\text { Verbal Fluency }\end{array}$ & Rey's Auditory, Memory Scanning Task \\
\hline Shenkin et al. [25] & $\begin{array}{l}\text { Verbal Fluency, Controlled Word Association, } \\
\text { Moray House Test, Raven's Progressive Matrices }\end{array}$ & Wechsler Memory Scale \\
\hline Baune et al. [26] & Stroop, Letter-Digit Substitution Task & 3-Word Recall \\
\hline Kim et al. [27] & $\begin{array}{l}\text { Boston Naming, Buccofacial Praxis Test, } \\
\text { Semantic Controlled Oral Word Association } \\
\text { Test, Stroop Color, Word Test }\end{array}$ & $\begin{array}{l}\text { Seoul Verbal Learning Test, Ray Complex Figure Test, } \\
\text { Delayed Recall and Recognition, Digit Span Tests }\end{array}$ \\
\hline Silbert et al. [28] & - & Delayed Story Recall \\
\hline McClleland et al. [21] & Digit-Symbol Substitution Task & - \\
\hline Wright et al. [29] & Color Trail $1 \& 2$ & - \\
\hline Kaplan et al. [30] & Stroop, Trail Making, CalCap & Repeated Battery for Neuropsychological Status \\
\hline Wakefield et al. [31] & Stroop, Trail Making 1 \& 2, CalCap & - \\
\hline O'Brien et al. [32] & Verbal Fluency, Trail Making $1 \& 2$ & Memory Component of CDR \\
\hline Smith et al. [14] & Letter Fluency, Trail Making 2 & Episodic Memory, Alpha Span Test \\
\hline Burns et al. [33] & $\begin{array}{l}\text { Trail Making } 1 \text { \& 2, Short Blessing Test, } \\
\text { Boston Naming }\end{array}$ & $\begin{array}{l}\text { Wechsler Memory Scale, Wechsler Adult } \\
\text { Intelligence Scale }\end{array}$ \\
\hline Ishii et al. [34] & $\begin{array}{l}\text { Verbal Fluency, Trail Making Test, } \\
\text { Benton's Visual Form Test }\end{array}$ & ADAS-Cog, 10 Word Recall, Digit Span Forward \\
\hline Tullberg et al. [35] & Verbal Fluency & $\begin{array}{l}\text { Wechsler Memory Scale, Word List Learning, } \\
\text { Digit Span Backward }\end{array}$ \\
\hline
\end{tabular}

analysis was on the distribution of WMLs in the internal and external capsule. They found that WMLs from both regions were significantly associated with cognitive performance of speed of memory retrieval and executive function/processing speed $(\mathrm{p}<0.05)$.

Smith et al. [14] analyzed WML distribution using PD, $\mathrm{T} 2$, and $\mathrm{T} 1$ scans in the whole brain of 147 older adults. The total volume of WMLs was associated with the cognitive performance of memory $(\mathrm{p}<0.01)$ and executive function $(\mathrm{p}=0.05)$. In the following locations, WMLs were significantly associated with memory: right inferior temporal-occipital, left temporal-occipital periventricular, and right parietal periventricular; and anterior limb of internal capsule. Also, WMLs in the following regions were significantly associated with executive function: the bilateral inferior frontal, temporal-occipital periventricular, right parietal periventricular, and prefrontal white matter; and the anterior limb of the internal capsule bilaterally.

\section{Studies that classified and compared subjects based on cognitive status}

Subcortical vs. periventricular WML

Among the studies that classified participants based on their cognitive status, two cross-sectional studies [33,34] compared the effects of subcortical and periventricular
WMLs. Burns et al. [33] included 88 non-demented participants (clinical dementia rating (CDR) score $=0$ ), 68 with early-stage $\mathrm{AD}$ (48 with very mild $\mathrm{AD}(\mathrm{CDR}=0.5)$, and 20 with mild $\mathrm{AD}(\mathrm{CDR}=1))$. White matter lesions were defined as T2 hyperintensities that were T1 hypointensities. Subcortical WMLs were rated in regions of frontal, parietal, temporal, and occipital lobes. Periventricular WMLs were rated in right and left frontal horns, posterior horns, and ventricular bodies. For nondemented participants, only associate memory was associated with periventricular WMLs $(\mathrm{p}<0.01)$. For participants with early-stage $\mathrm{AD}$, memory and executive function/ processing speed were associated with both periventricular and subcortical WMLs $(\mathrm{p}<0.05)$.

Ishii et al. [34] detected WMLs based on T2 hyperintensities. Sample of 453 older adults were categorized into two groups of $\mathrm{CDR}=0$ and $\mathrm{CDR}=0.5$. Anterior and posterior periventricular WMLs, as well as left and right subcortical WMLs were segmented. The results suggested that, for $\mathrm{CDR}=0$ group, anterior periventricular WMLs and a test of executive function/processing speed were significantly correlated $(\mathrm{p}=0.001)$.

\section{Regional WML}

The last study [35] detected WMLs based on T1 and T2 scans. They categorized 78 older adults into three 
Table 5 Association between the structural location of white matter lesion (i.e., subcortical, periventricular, or regional) with two domains of cognitive function (i.e., memory and executive function/processing speed)

\begin{tabular}{ll}
\hline Reference & Association \\
\hline Groot et al. et al. [24] & Controlled for subcortical, periventricular WMLs were associated with memory and executive function/processing speed. \\
Shenkin et al. [25] & Subcortical and periventricular WMLs were not associated with any of the cognitive measurements. \\
Baune et al. [26] & Subcortical WMLs were associated with memory. \\
& As a subgroup of subcortical WMLs, infarction lesions were associated with executive function/processing speed. \\
& Periventricular WMLs were not associated with any of the cognitive functions.
\end{tabular}

Kim et al. [27]

Only periventricular WML was significantly correlated with memory and executive function/processing speed, when both the periventricular and subcortical WMLs were entered simultaneously into the regression model.

Silbert et al. [28] Change in subcortical WMLs (excluding infarction lesions) was associated with memory decline. This association was not true for periventricular WMLs.

McClleland et al. [21] White matter lesions were associated with executive function/processing speed, in all white matter regions of cerebrum, cerebellum, and basal ganglia.

Wright et al. [29] Subcortical WMLs (including infarction lesions) were associated with executive function/processing speed, in regions of frontal and deep white matter.

Kaplan et al. [30] White matter lesions were associated with memory and executive function/processing speed, in frontal regions.

Wakefield et al. [31] White matter lesions were associated with executive function/processing speed in white matter regions of posterior corona radiata and splenium of corpus callosum.

O'Brien et al. [32]

White matter lesions were associated with speed of memory retrieval and executive function/processing speed.

Smith et al. [14]

White matter lesions were associated with memory and executive function/processing speed. White matter lesions in the following locations were significantly associated with memory: right inferior temporal-occipital, left temporal-occipital periventricular, and right parietal periventricular white matter; and anterior limb of internal capsule. Also, WMLs in the following regions were significantly associated with executive function: the bilateral inferior frontal, temporal-occipital periventricular, right parietal periventricular, and prefrontal white matter; and the anterior limb of the internal capsule bilaterally.

Burns et al. [33]

For non-demented participants, only associate memory was associated with periventricular WMLs. For participants with early-stage Alzheimer's Disease (AD), memory and executive function/processing speed were associated with both periventricular and subcortical WMLs.

Ishii et al. [34]

For $C D R=0$ group, anterior periventricular WML and a test of executive function/processing speed were significantly correlated.

Tullberg et al. [35] In non-demented individuals, increased volumes of frontal (specifically prefrontal and dorsolateral), parietal, and occipital WML were separately associated with lower executive function/processing speed scores.

Frontal WMLs were also associated with reduced memory function in non-demented group. No association was found for individuals with dementia.

Abbreviations: $W M L=$ White Matter Lesion; $C D R=$ Clinical Dementia Rating Scale

cognitive groups: normal $(\mathrm{CDR}=0)$, cognitively impaired but not demented $(\mathrm{CDR}=0.5)$, and demented $(\mathrm{CDR} \geq 1)$, either by $\mathrm{AD}$ or vascular dementia. WMLs were analyzed in regions of orbitofrontal, prefrontal, dorsolateral frontal, parietal, and occipitotemporal. In non-demented individuals, increased volumes of frontal (specifically, prefrontal and dorsolateral), parietal, and occipital WML were separately associated with lower executive function/processing speed scores $(\mathrm{p}<0.05)$. Frontal WMLs were also associated with reduced memory function in non-demented group $(\mathrm{p}<0.05)$. No association was found for individuals with dementia.

\section{Table 6 The most commonly-used neuropsychological} tests in the included studies

\begin{tabular}{ll}
\hline Executive Function & Trail-Making Test, Stroop Test, Verbal Fluency Test \\
Memory & Wechseler Memory Scale, Word Recall Test \\
\hline
\end{tabular}

\section{Quality assessment}

The quality assessment results for each of the four questions are presented in Table 1:1) in seven studies, WML identification is done by a radiologist/physician, while the remaining used automatic methods; 2) all the articles employed standard methods for cognitive assessment; 3 ) none of the studies provided sample size calculation; and 4) the statistical analyses of twelve studies included age or education as confounders.

\section{Discussion}

\section{Subcortical vs. periventricular WMLs}

Based on their proximity to ventricles, WMLs were classified as subcortical or periventricular in seven studies [24-28,33,34]. The results show that more studies have found an association between periventricular WMLs with the cognitive domain of executive function, than subcortical WMLs. 
Subcortical WMLs are believed to primarily disrupt short connections, and thus impairing cognitive performance supported by the specific brain region [24]. For example, dexterous hand and arm movements are generally thought to be primarily supported by the motor cortex. Therefore, subcortical WMLs in this specific region can result in reduced performance in hand and arm dextrous movements [36]. In contrast, periventricular WMLs disrupt longer connections to spatially distant cortical areas, and thus can cause cognitive performance decline in multiple domains [24,27]. For example, executive function tasks typically used in research experiments depend on multiple brain regions (i.e., frontal and non-frontal) which are not necessarily located spatially close to each other [37]. Therefore, any disruption in long white matter tracts traversing from periventricular areas may initially reduce the axonal transmission speed [38], and later cause impaired executive function. In summary, cognitive function depends on intact connections within subcortical areas and between cortical and subcortical structures, and any disruption in these connections may impair cognitive function.

We categorized all included studies into two major cognitive domains which are sensitive to aging: 1 ) memory; or 2) executive function/processing speed. The latter category was a combination of two cognitive domains based on the idea that they are not mutually exclusive, and one needs to control for their mutual relationship before examining their unique effects [39].

For memory, out of seven studies, three studies $[24,27,33]$ found a significant association between periventricular WMLs and memory performance, two studies [26,28] found a significant association between subcortical WMLs and memory performance, and two studies $[25,34]$ did not find any association.

For executive function/processing speed, out of six studies, three studies $[24,27,34]$ found a significant association between periventricular WMLs and executive function/ processing speed, while only one study [26] found a significant association between subcortical WMLs and executive function/processing speed. Two studies [25,33] did not find any association.

Thus, our overall results show that greater number of studies found an association between cognitive impairment (in both domains of memory and executive function/processing speed) and periventricular WMLs, compared with subcortical WMLs. Moreover, greater number of studies showed an association between impairment in the domain of executive function/processing speed with periventricular WMLs, compared to subcortical WMLs.

As highlighted earlier, periventricular WMLs may impact multiple domains of cognition because they disrupt distant connections. Hence, our findings concur with the general knowledge that the domain of executive function/processing speed may depend on multiple brain regions and spatially distant connections $[37,40]$.

\section{Regional WML}

Seven studies [14,21,29-32,35] investigated regional WMLs. No common pattern was evident secondary to the heterogeneity of regions studied.

The following regions demonstrated significant associations between WMLs and cognitive function: cerebral white matter, cerebellar white matter, and basal ganglia [21]; frontal (dorsolateral frontal and prefrontal) [29-31], parietal, occipital, and temporal lobes [29,31,35]; internal and external capsule [32]; posterior corona radiata, and splenium corpus callosum [31]. This systematic review provides researchers with a summary set of brain regions in which an association have been found between WMLs and cognitive performance. To better understand the role of anatomical location in the association between WML and cognitive function, future studies should examine the spatial distribution of WMLs on the whole brain, or specific set of brain regions identified in this review as being highly associated with cognitive dysfunction.

\section{Limitations}

The discrepancies between the results may be due to the heterogeneous study methodologies and the quality of included studies.

\section{Different MRI sequences, WML quantification methods, and neuropsychological batteries}

The included studies were heterogeneous in MRI sequences for WML detection (i.e., PD, T1, T2, or FLAIR), WML quantification method (i.e., scoring or volume measurements), and components of neuropsychological batteries. This likely contributed to variability in our results.

Moreover, two different methods were used for WML quantification: 1) scoring [24,25,33,34]; and 2) volume measurement [26-28]. Scoring measures are usually done manually, and show a higher accuracy for selection of subtle WMLs, compared to automatic volumetric methods. However, these methods vary significantly in terms of lesion classification and severity scoring. Moreover, each scoring method has its own specific limitations.

For WML volume measurement, there are two steps. The first step is identifying lesions, which can be done either manually by an expert radiologist or automatically. After the WMLs are identified manually or automatically, one can proceed to the second step, which is measuring WML volumes automatically. It has been shown that both scoring and volumetric quantification methods are reliable for measuring WML load [41,42]. However, periventricular and subcortical WMLs quantified by these two 
quantification methods are differently associated with cognitive function [42]. Out of three studies which used volume measurement, two studies [26,28] showed a significant association between the subcortical WMLs and cognitive performance. Out of four studies which used scoring, three $[24,33,34]$ showed a significant association between periventricular WMLs and cognitive performance. These results suggest that scoring might have biased the results toward periventricular WMLs. Conversely, volume measurement might be problematic for periventricular WMLs due to their similar appearance to CSF on some MRI sequences (e.g., T2 or T1) [43].

\section{Modifying effect of cardiovascular risk factors}

There is a growing recognition that WMLs are associated with age and cardiovascular risk factors [8,44]. However, all but one included study [21] considered the modifying effect of cardiovascular risk factors in the statistical analysis. We recommend that future studies consider including cardiovascular risk factors in their analysis.

\section{Quality of studies and lack of sample size calculation}

One study [25] did not demonstrate a significant association between any type of WMLs and any of the cognitive tasks. Based on our quality assessment, this study is the only study categorizing WMLs locations as subcortical and periventricular that did not consider age or education as potential confounders. Therefore, we concluded that this study did not provide strong evidence for the lack of correlation between WMLs and cognitive function.

Moreover, the lack of sample size calculations in all of the included studies might have resulted in possible type II errors. However, we do recognize that the lack of sample size calculations may be due to the dearth of data in this research area [45].

\section{Conclusions}

This study provides the first in depth analysis of brain regions where an association between WML location and cognitive decline has been found in older adults. Specifically, studies that considered periventricular versus subcortical WMLs suggest that, compared with subcortical WMLs, periventricular WMLs may have a greater negative impact on cognitive performance. Moreover, periventricular WMLs appear to be more associated to the domain of executive function/processing speed, than to the domain of memory. To further clarify the association of cognitive function with WML locations, we suggest that future studies consider spatial distribution of WMLs on the whole brain.

We did not proceed to a meta-analysis of the results, primarily because of the small number of studies systematically found on this topic. Moreover, the neuropsychological batteries used for assessing cognitive status, the WML quantification method, and MRI sequences used for WML detection varied vastly between studies. Thus, it was not feasible to conduct a meta-analysis.

\section{Source of funding}

This work was supported by the Canadian Stroke Network, the Heart and Stroke Foundation of Canada, and the Canadian Institutes of Health Research (MOB-93373) to TLA. TLA is a Canada Research Chair (Tier II) in Physical Activity, Mobility, and Cognitive Neuroscience, a Michael Smith Foundation for Health Research Scholar, a Canadian Institutes of Health Research New Investigator, and a Heart and Stroke Foundation of Canada's Henry JM Barnett's Scholarship recipient. JCD is a Michael Smith Foundation for Health Research Post-Doctoral Fellow and a Canadian Institutes of Health Research Post-Doctoral Fellow. NB is a Heart and Stroke Foundation of Canada Doctoral Trainee.

\section{Competing interests}

The authors declare that they have no competing interests.

Authors' contribution

All authors participated, read, and approved the final manuscript.

\section{Author details}

'Department of Physical Therapy, University of British Columbia, 212-2177 Wesbrook Mall, Vancouver, BC V6T 1Z3, Canada. ${ }^{2}$ Centre for Clinical Epidemiology and Evaluation, University of British Columbia, 212-2177 Wesbrook Mall, Vancouver, BC V6T 1Z3, Canada. ${ }^{3}$ Department of Radiology, University of British Columbia, 212-2177 Wesbrook Mall, Vancouver, BC V6T 1Z3, Canada. ${ }^{4}$ Department of Psychology, University of British Columbia, 212-2177 Wesbrook Mall, Vancouver, BC V6T 1Z3, Canada. ${ }^{5}$ Brain Research Centre, University of British Columbia, 212-2177 Wesbrook Mall, Vancouver, BC V6T 1Z3, Canada. ${ }^{6}$ Centre for Hip Health and Mobility, Vancouver Coastal Health Research Institute, 212-2177 Wesbrook Mall, Vancouver, BC V6T 1Z3, Canada.

Received: 28 March 2012 Accepted: 12 October 2012

Published: 30 October 2012

\section{References}

1. Kinsella K: Demographic dimensions of global aging. J Fam Issues 2000, 21(5):541-558.

2. Park DC, Reuter-Lorenz P: The adaptive brain: aging and neurocognitive scaffolding. Annu Rev Psychol 2009, 60:173-196.

3. Haug H, Eggers R: Morphometry of the human cortex cerebri and corpus striatum during aging. Neurobiol Aging 1991, 12(4):336-338. discussion 352-335.

4. Adolfsson R, Gottfries CG, Roos BE, Winblad B: Post-mortem distribution of dopamine and homovanillic acid in human brain, variations related to age, and a review of the literature. J Neural Transm 1979, 45(2):81-105.

5. de Keyser J, De Backer JP, Vauquelin G, Ebinger G: The effect of aging on the D1 dopamine receptors in human frontal cortex. Brain Res 1990, 528(2):308-310.

6. Breteler MM, van Swieten JC, Bots ML, Grobbee DE, Claus JJ, van den Hout $J$ H, van Harskamp F, Tanghe HL, de Jong PT, van Gijn J, et al: Cerebral white matter lesions, vascular risk factors, and cognitive function in a population-based study: the rotterdam study. Neurology 1994, 44(7):1246-1252.

7. Lindgren A, Roijer A, Rudling O, Norrving B, Larsson EM, Eskilsson J, Wallin L, Olsson B, Johansson BB: Cerebral lesions on magnetic resonance imaging, heart disease, and vascular risk factors in subjects without stroke. A population-based study. Stroke 1994, 25(5):929-934. 
8. Kuo HK, Lipsitz LA: Cerebral white matter changes and geriatric syndromes: is there a link? J Gerontol A Biol Sci Med Sci 2004, 59(8):818-826.

9. Galluzzi S, Lanni C, Pantoni L, Filippi M, Frisoni GB: White matter lesions in the elderly: pathophysiological hypothesis on the effect on brain plasticity and reserve. J Neurol Sci 2008, 273(1-2):3-9.

10. Frisoni GB, Galluzzi S, Pantoni L, Filippi M: The effect of white matter lesions on cognition in the elderly-small but detectable. Nat Clin Pract Neurol 2007, 3(11):620-627.

11. Pantoni L, Garcia JH: Pathogenesis of leukoaraiosis: a review. Stroke 1997, 28(3):652-659.

12. Jokinen H, Kalska H, Ylikoski R, Madureira S, Verdelho A, Gouw A, Scheltens $P$, Barkhof F, Visser MC, Fazekas F: MRI-defined subcortical ischemic vascular disease: baseline clinical and neuropsychological findings. Cerebrovasc Dis 2009, 27(4):336-344.

13. Pantoni L: Pathophysiology of age-related cerebral white matter changes. Cerebrovasc Dis 2002, 13(2):7-10.

14. Smith EE, Salat DH, Jeng J, McCreary CR, Fischl B, Schmahmann JD, Dickerson BC, Viswanathan A, Albert MS, Blacker D, et al: Correlations between MRI white matter lesion location and executive function and episodic memory. Neurology 2011, 76(17):1492-1499.

15. Pantoni L, Poggesi A, Inzitari D: Cognitive decline and dementia related to cerebrovascular diseases: some evidence and concepts. Cerebrovasc Dis 2009, 27(1):191-196.

16. Pantoni L, Poggesi A, Inzitari D: The relation between white-matter lesions and cognition. Curr Opin Neurol 2007, 20(4):390-397.

17. Kim SH, Park JS, Ahn HJ, Seo SW, Lee JM, Kim ST, Han SH, Na DL: Voxelbased analysis of diffusion tensor imaging in patients with subcortical vascular cognitive impairment: correlates with cognitive and motor deficits. J Neuroimaging 2011, 21(4):317-324.

18. Desmond DW: Cognition and white matter lesions. Cerebrovasc Dis 2002, 13(2):53-57.

19. Liberati A, Altman DG, Tetzlaff J, Mulrow C, Gotzsche PC, loannidis JP, Clarke M, Devereaux PJ, Kleijnen J, Moher D: The PRISMA statement for reporting systematic reviews and meta-analyses of studies that evaluate health care interventions: explanation and elaboration. J Clin Epidemio/ 2009 62(10):e1-e34.

20. Gunning-Dixon FM, Raz N: The cognitive correlates of white matter abnormalities in normal aging: a quantitative review. Neuropsychology 2000, 14(2):224-232

21. McClelland RL, Kronmal RA, Bryan RN, Manolio TA, Herskovits E, Kuller LH O'Leary DH: Neurologic correlates of infarction-like lesion location on magnetic resonance imaging in the cardiovascular health study. J Stroke Cerebrovasc Dis 2000, 9(5):218-228.

22. Filippi M, Falini A, Arnold DL, Fazekas F, Gonen O, Simon JH, Dousset V, Savoiardo M, Wolinsky JS: Magnetic resonance techniques for the in vivo assessment of multiple sclerosis pathology: consensus report of the white matter study group. J Magn Reson Imaging 2005, 21(6):669-675.

23. McAusland J, Tam R, Wong E, Riddehough A, Li D: Optimizing the use of radiologist seed points for improved multiple sclerosis lesion segmentation. IEEE Trans Biomed Eng 2010,

24. de Groot JC, de Leeuw FE, Oudkerk M, van Gijn J, Hofman A, Jolles J, Breteler MM: Cerebral white matter lesions and cognitive function: the rotterdam scan study. Ann Neurol 2000, 47(2):145-151.

25. Shenkin SD, Bastin ME, Macgillivray TJ, Deary IJ, Starr JM, Rivers CS, Wardlaw JM: Cognitive correlates of cerebral white matter lesions and water diffusion tensor parameters in community-dwelling older people. Cerebrovasc Dis 2005, 20(5):310-318.

26. Baune BT, Roesler A, Knecht S, Berger K: Single and combined effects of cerebral white matter lesions and lacunar infarctions on cognitive function in an elderly population. J Gerontol A Biol Sci Med Sci 2009, 64(1):118-124

27. Kim JH, Hwang KJ, Lee YH, Rhee HY, Park KC: Regional white matter hyperintensities in normal aging, single domain amnestic mild cognitive impairment, and mild Alzheimer's disease. J Clin Neurosci 2011, 18(8):1101-1106.

28. Silbert LC, Nelson C, Howieson DB, Moore MM, Kaye JA: Impact of white matter hyperintensity volume progression on rate of cognitive and motor decline. Neurology 2008, 71(2):108-113.

29. Wright CB, Festa JR, Paik MC, Schmiedigen A, Brown TR, Yoshita M, DeCarli C, Sacco R, Stern Y: White matter hyperintensities and subclinical infarction: associations with psychomotor speed and cognitive flexibility. Stroke 2008, 39(3):800-805.

30. Kaplan RF, Cohen RA, Moscufo N, Guttmann C, Chasman J, Buttaro M, Hall $\mathrm{CH}$, Wolfson L: Demographic and biological influences on cognitive reserve. J Clin Exp Neuropsychol 2009, 31(7):868-876.

31. Wakefield DB, Moscufo N, Guttmann CR, Kuchel GA, Kaplan RF, Pearlson G, Wolfson L: White matter hyperintensities predict functional decline in voiding, mobility, and cognition in older adults. J Am Geriatr Soc 2010, 58(2):275-281.

32. O'Brien JT, Wiseman R, Burton EJ, Barber B, Wesnes K, Saxby B, Ford GA: Cognitive associations of subcortical white matter lesions in older people. Ann N Y Acad Sci 2002, 977:436-444

33. Burns JM, Church JA, Johnson DK, Xiong C, Marcus D, Fotenos AF, Snyder $A Z$, Morris JC, Buckner RL: White matter lesions are prevalent but differentially related with cognition in aging and early Alzheimer disease. Arch Neurol 2005, 62(12):1870-1876.

34. Ishii $H$, Meguro $K$, Yamaguchi $S$, Ishikawa $H$, Yamadori A: Prevalence and cognitive performances of vascular cognitive impairment no dementia in Japan: the Osaki-Tajiri Project. Eur J Neurol 2007, 14(6):609-616.

35. Tullberg M, Fletcher E, DeCarli C, Mungas D, Reed BR, Harvey DJ, Weiner MW, Chui HC, Jagust WJ: White matter lesions impair frontal lobe function regardless of their location. Neurology 2004, 63(2):246-253.

36. Darling WG, Pizzimenti MA, Rotella DL, Peterson CR, Hynes SM, Ge J, Solon K, McNeal DW, Stilwell-Morecraft KS, Morecraft RJ: Volumetric effects of motor cortex injury on recovery of dexterous movements. Exp Neurol 2009, 220(1):90-108.

37. Alvarez JA, Emory E: Executive function and the frontal lobes: a metaanalytic review. Neuropsychol Rev 2006, 16(1):17-42.

38. van den Heuvel DM, ten Dam VH, de Craen AJ, Admiraal-Behloul F, Olofsen H, Bollen EL, Jolles J, Murray HM, Blauw GJ, Westendorp RG, et al: Increase in periventricular white matter hyperintensities parallels decline in mental processing speed in a non-demented elderly population. J Neurol Neurosurg Psychiatry 2006, 77(2):149-153.

39. Albinet $\mathrm{CT}$, Boucard $\mathrm{G}$, Bouquet $\mathrm{CA}$, Audiffren M: Processing speed and executive functions in cognitive aging: How to disentangle their mutual relationship? Brain Cogn 2012, 79(1):1-11.

40. Carpenter PA, Just MA, Reichle ED: Working memory and executive function: evidence from neuroimaging. Curr Opin Neurobiol 2000, 10(2):195-199.

41. Gouw A, Van Der Flier W, Van Straaten E, Pantoni L, Bastos-Leite A, Inzitari $D$, Erkinjuntti T, Wahlund L, Ryberg C, Schmidt R: Reliability and sensitivity of visual scales versus volumetry for evaluating white matter hyperintensity progression. Cerebrovasc Dis 2008, 25(3):247-253.

42. Tiehuis A, Vincken K, Mali WPTM, Kappelle L, Anbeek P, Algra A, Biessels G: Automated and visual scoring methods of cerebral white matter hyperintensities: relation with age and cognitive function. Cerebrovasc Dis 2008, 25(1-2):59-66.

43. Sajja BR, Datta S, He R, Mehta M, Gupta RK, Wolinsky JS, Narayana PA: Unified approach for multiple sclerosis lesion segmentation on brain MRI. Ann Biomed Eng 2006, 34(1):142-151.

44. Debette S, Markus HS: The clinical importance of white matter hyperintensities on brain magnetic resonance imaging: systematic review and meta-analysis. BMJ 2010, 341:C3666.

45. Abdul Latif L, Daud Amadera JE, Pimentel D, Pimentel T, Fregni F: Sample size calculation in physical medicine and rehabilitation: a systematic review of reporting, characteristics, and results in randomized controlled trials. Arch Phys Med Rehabil 2011, 92(2):306-315.

doi:10.1186/1471-2377-12-126

Cite this article as: Bolandzadeh et al:: The association between cognitive function and white matter lesion location in older adults: a systematic review. BMC Neurology 2012 12:126. 